\title{
EDITORIAL
}

\section{Targeting levels and functions of blood lipids in the prevention of CVD}

D yslipidemia is a major cause of cardiovascular disease (CVD), and high total cholesterol levels account for one-third of deaths associated with ischemic heart disease (WHO. Global health risks: mortality and burden of disease attributable to selected major risks. WHO Press, Geneva, 2009). Accumulating data on risk prediction and mechanisms of disease have uncovered multiple potential targets for treatment of patients with dyslipidemia. Given the considerable global impact of this condition, and the increasing number of therapeutic options available, we have commissioned a group of articles that focus on the latest knowledge about blood lipids and their link with CVD risk. These articles will be published in two print issues of Nature Reviews Cardiology, the first of which (this issue) highlights the most-recent findings on lipid parameters that should be used to assess CVD risk, genetic determinants of lipid levels linked with CVD and vascular health, and biological mechanisms of disease protection. Our May print issue will include three Review articles discussing the latest findings on optimum management strategies for patients with dyslipidemia.

LDL-cholesterol levels are the most-commonly measured lipid parameter for the prediction of CVD risk and the identification of patients who would probably benefit from lipid-lowering therapy. Nevertheless, other lipid-related measurements are being found to provide additional predictive value. In a Review published in this issue of Nature Reviews Cardiology, John Kastelein and colleagues discuss the measurement of various lipoprotein-lipid parameters and the evidence supporting the argument that some of these parameters should be more-routinely assessed.

Unlike many nongenetic risk factors, a patient's genotype does not vary over the course of their life. Because of this characteristic, and given its low cost and high fidelity, genetic testing is an attractive tool for CVD risk prediction, particularly in young individuals. In this issue, Aroon Hingorani, Steve Humphries and colleagues examine the potential applications and challenges of using genetic information about lipid risk factors for CVD prediction.

Although LDL-cholesterol levels are the mostcommon lipid parameter assessed, HDL cholesterol has received a lot of attention in the past few years as a result of increased knowledge of its atheroprotective effects. The importance of HDL function, in addition to its levels, is becoming increasingly clear. Furthermore,

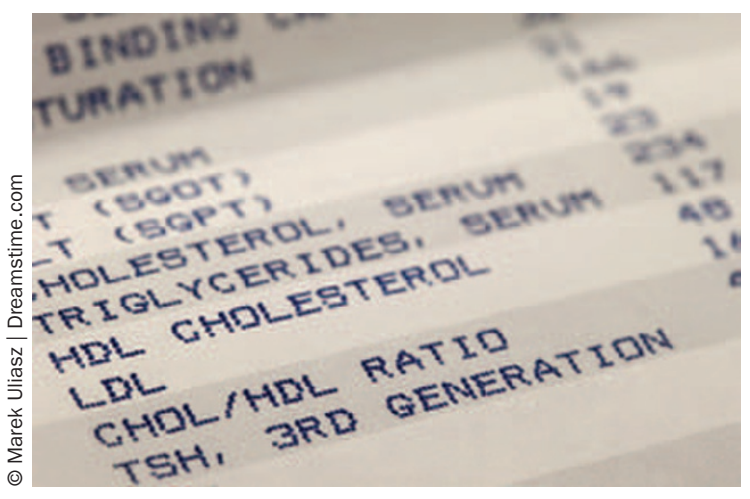

whilst HDL is well known to be involved in reverse cholesterol transport, data on other atheroprotective roles, including inhibition of monocyte migration, increased NO production, inhibition of the coagulation cascade, and antioxidative activity, are emerging. Mohamad Navab, Alan Fogelman and colleagues focus on the mechanisms by which HDL protects against CVD in their Review article, and highlight potential approaches to assess and modulate HDL function in the clinical setting.

Statins are first-line therapy for reducing LDLcholesterol levels in most patients and numerous statins are currently on the market. Other agents, such as niacin, bile-acid sequestrants, and ezetimibe, are also available for patients with elevated LDL-cholesterol levels. However, high-risk patients might require reductions in LDL-cholesterol levels beyond those that can be achieved with available drugs, and investigational agents that reduce the concentration of LDL cholesterol by other mechanisms are currently under development. In the first of three articles on therapy for dyslipidemia, which will be published in the May print issue of Nature Reviews Cardiology, Ariel Brautbar and Christie Ballantyne examine currently available drugs and experimental agents designed to achieve 'optimal' LDL-cholesterol levels.

Although most guidelines highlight reduction of LDL-cholesterol levels as the primary target of therapy, multiple therapeutic studies over the past decade or so have focussed on HDL cholesterol. Excitement around targeting HDL cholesterol was somewhat tempered in 2007 by the adverse effects associated with torcetrapib in the ILLUMINATE trial (Barter, P J. N. Engl. J. Med. 357, 2109-2122; 2007), but has been reignited with
Bryony M. Mearns is the Chief Editor of Nature Reviews Cardiology.

Competing interests The author declares no competing interests. 
the publication of the phase III safety trial of anacetrapib (DEFINE; Cannon, C. P. et al. N. Engl. J. Med. 363, 2406-2415; 2010) in late 2010. In the May print issue of Nature Reviews Cardiology, Emil deGoma and Daniel Rader discuss the mechanisms of HDLtargeted drugs and summarize the development of these pharmacotherapies in preclinical and clinical studies.

Patients with diabetes mellitus are at increased risk of coronary heart disease compared with the general population, and multiple trials over the past few years, including the phase III ACCORD trial (The ACCORD study group. N. Engl. J. Med. 362, 1563-1574; 2010), have addressed treatment in this particular patient subgroup. In the May print issue of the journal, John Betteridge focuses on patients with diabetes mellitus and discusses available data from randomized controlled trials of statins and other lipid-targeting pharmaceutical agents that provide a framework to guide primary and secondary prevention of CVD in this patient population.

We hope that you read our round-up of the latest developments in research of blood lipids and their role in CVD risk with interest, and consider this article collection a useful resource.

doi:10.1038/nrcardio.2011.42 